\title{
In-House Digital Workflow for the Management of Acute Mandible Fractures
}

Jeffrey S. Marschall DMD, MD, MS ${ }^{1}$, Vinicius Dutra DDS, MBA, $\mathrm{PhD}^{2}$, Robert L. Flint DMD, MD², George M. Kushner DMD, MD², Brian Alpert DDS ${ }^{5}$, William Scarfe BDS ${ }^{6}$, Bruno Azevedo DDS, MS ${ }^{7}$.

1. Resident, Department of Oral and Maxillofacial Surgery, University of Louisville School of Dentistry, Louisville, KY

2. Visiting Scholar, Department of Diagnosis and Oral Health, University of Louisville School of Dentistry, Louisville, KY

3. Assistant Professor, Department of Oral and Maxillofacial Surgery, University of Louisville School of Dentistry Louisville, KY

4. Professor and Program Director, Advanced Education Program in Oral and Maxillofacial Surgery, University of Louisville School of Dentistry, Louisville, KY

5. Professor, Department of Oral and Maxillofacial Surgery, University of Louisville School of Dentistry, and Chief, Oral and Maxillofacial Surgery and Dentistry, University of Louisville Hospital, Louisville, KY

6. Professor, Department of Diagnosis and Oral Health, University of Louisville School of Dentistry, Louisville, KY

7. Associate Professor, Department of Diagnosis and Oral Health, University of Louisville School of Dentistry, Louisville, KY

Dr Dutra is currently affiliated with the Indiana University School of Dentistry, Indianapolis, IN.

Conflict of Interest Disclosures: None of the authors have any relevant financial relationship(s) with a commercial interest.

This is the author's manuscript of the article published in final edited form as:

Marschall, J. S., Dutra, V., Flint, R. L., Kushner, G. M., Alpert, B., Scarfe, W., \& Azevedo, B. (2019). In-House Digital Workflow for the Management of Acute Mandible Fractures. Journal of Oral and Maxillofacial Surgery, $77(10)$, 2084.e1-2084.e9. https://doi.org/10.1016/j.joms.2019.05.027 
Computer-aided design and additive manufacturing are revolutionizing oral and maxillofacial surgery. Current methods use virtual surgical planning sessions and custom plate milling via third-party vendors, which is costly and time-consuming, negating the effectiveness in acute facial trauma. This technical note describes a state-of-the-art inhouse expedited digital workflow for computer-aided virtual fracture reduction, 3dimensional printing, and preoperative reconstruction plate adaptation for the management of an acute mandible fracture. This process uses the computed tomographic scan a patient receives in the emergency department or clinic. The DICOM (Digital Imaging and Communications in Medicine) data are transferred into US Food and Drug Administration-approved software, in which the fracture is segmented and virtually reduced based on condylar position, midline symmetries, and occlusion if present. The reduced mandible is then printed, which serves as a template for preoperative reconstruction plate adaptation. This method facilitates a virtually reduced fractured mandible, 3-dimensionally printed model, and ideally adapted plates ready for sterilization before surgery within 2 hours after DICOM upload.

Traditional repair of maxillofacial trauma patients has centered on the ability to establish correct preinjury occlusion via intermaxillary fixation (IMF), allowing for open reductioninternal fixation (ORIF) with titanium reconstruction plates contoured during surgery. Subjective determination of intraoperative occlusion and reconstruction plate contouring are time-consuming and dependent on surgeon skill. Traditional ORIF has undoubtedly stood the test of time; however, computer-aided design (CAD) and computer-aided manufacturing (CAM) are providing methods that are changing the way oral and maxillofacial surgery is completed. $\underline{1}, \underline{2}, \underline{3}, \underline{4}$ Considering that surgeons now have the ability to preplan and enhance execution, many are using CAD-CAM as their go-to method in orthognathic surgery, craniofacial surgery, and microvascular surgery. $\underline{5}, \underline{6}, \underline{7}$ Operating room (OR) costs have and will always be a major concern; preoperative plate contouring has the ability to reduce OR time and costs, adding to its allure. - Even though CAD-CAM technologies are being used regularly in many areas of craniomaxillofacial surgery, literature focusing on acute trauma is sparse. 
The use of CAD-CAM as a modality in the treatment of acute facial trauma has been documented mostly in orbital and midface trauma. $\stackrel{-}{ }$ However, a handful of studies are starting to emerge describing CAD-CAM in the management of acute mandible fractures via online virtual surgical planning (VSP) sessions and custom-milled prefabricated plates. ${ }^{4}$ The ability to have a VSP session in which fractures can be virtually reduced and custom plates fabricated cannot be underestimated; unfortunately, substantial cost and lead time are required for this technique. Indeed, it can take 7 to 14 days to have a VSP session and delivery of a custom-milled plate, negating the effectiveness to ideally treat acute facial injuries. Several studies have described a less expensive alternative using on-site commercially available 3-dimensional (3D) printers.10, 11 This method requires the fabrication of 3-dimensionally printed models from in-office printers and either preoperative $\frac{11}{11}$ or intraoperative $\frac{10}{10}$ plate bending. A study showed that preoperative plate bending has the added benefit of decreasing OR time and consequently costs $\frac{11}{1}$; however, this study required manual post-fabrication manipulation to anatomically reduce the fracture. Elegbede et al 10 described an in-office method to virtually reduce simple mandible fractures based on mirror imaging of the contralateral side but contouring of the reconstruction plate to the model was performed intraoperatively, negating the cost savings of preoperative plate adaptation. It is important to note that 3D Slicer was used in this study, which is currently not approved by the US Food and Drug Administration (FDA) for patient use in the United States. Both of these studies used slow 3D printers.

We describe a novel technique that merges the benefits of having an online VSP session with on-site 3D model fabrication. Specifically, this technical note describes in-house virtual fracture reduction based on occlusion (when available), anatomic midlines, and condyle position, followed by expedited ( $<1.5$ hours) 3D printing and preoperative plate adaptation for state-of-the art treatment of acute mandible fractures. 


\section{CASE REPORT}

A 66-year-old female patient was involved in a high-speed motor vehicle accident, which resulted in left and right mandibular body fractures. Other injuries included a subarachnoid hemorrhage. The patient received a standard maxillofacial computed tomography (CT) scan (1.5-mm cuts acquired on Siemens Edge 128; Siemens, Erlangen, Germany) in our university's emergency department (Fig 1A), whereupon the oral and maxillofacial surgery service was consulted for the management of the facial injury. After the consultation was completed, the DICOM (Digital Imaging and Communications in Medicine) files from the CT scanner were transferred to a compact disc. The DICOM files were imported into Mimics software (Materialise, Leuven, Belgium) with preferably soft tissue reconstructions (Fig 1). Within the "segmentation tab," a custom threshold mask was created for the patient (Fig 1B). The maxilla, mandible, and any bone fragments were separated using the "split mask" function (Fig 1C). The segmentation was finished using the "multiple slice edit tool" using the anatomy as a reference in each slice of the scan (Fig 1D). The segmented mandible fracture fragments were then converted into individual objects using the "calculate" tool. After the segmentation was completed, computer-aided virtual reduction was completed ( $\mathrm{Fig} 2)$. The first step was to ensure that the mandibular condyles were seated within the mandibular fossa appropriately. This was completed using the "rotate" and "move" tools; to ensure proper placement, the condyle and fossa must be visible in the axial, coronal, and sagittal slices (Fig 2A). Once this was completed, the mandible fracture bone fragments can be aligned. When applicable, "virtual" IMF can be completed using the occlusion as a guide ( $\left.\mathrm{Fig}_{2} \mathrm{~B}\right)$. It is our experience that the $\mathrm{CT}$ images of the occlusion are enough to grossly virtually reduce the fractured segments. If the patient is edentulous or functionally edentulous, anatomic reduction is completed using the same rotate and move tools, as in this case. After anatomic virtual reduction, mandibular symmetry was assessed using anatomic landmarks such as the midline of the nasal bones, intercondylar distance, and mental protuberance. If dentition is present, dental midlines can be used as a reference. 


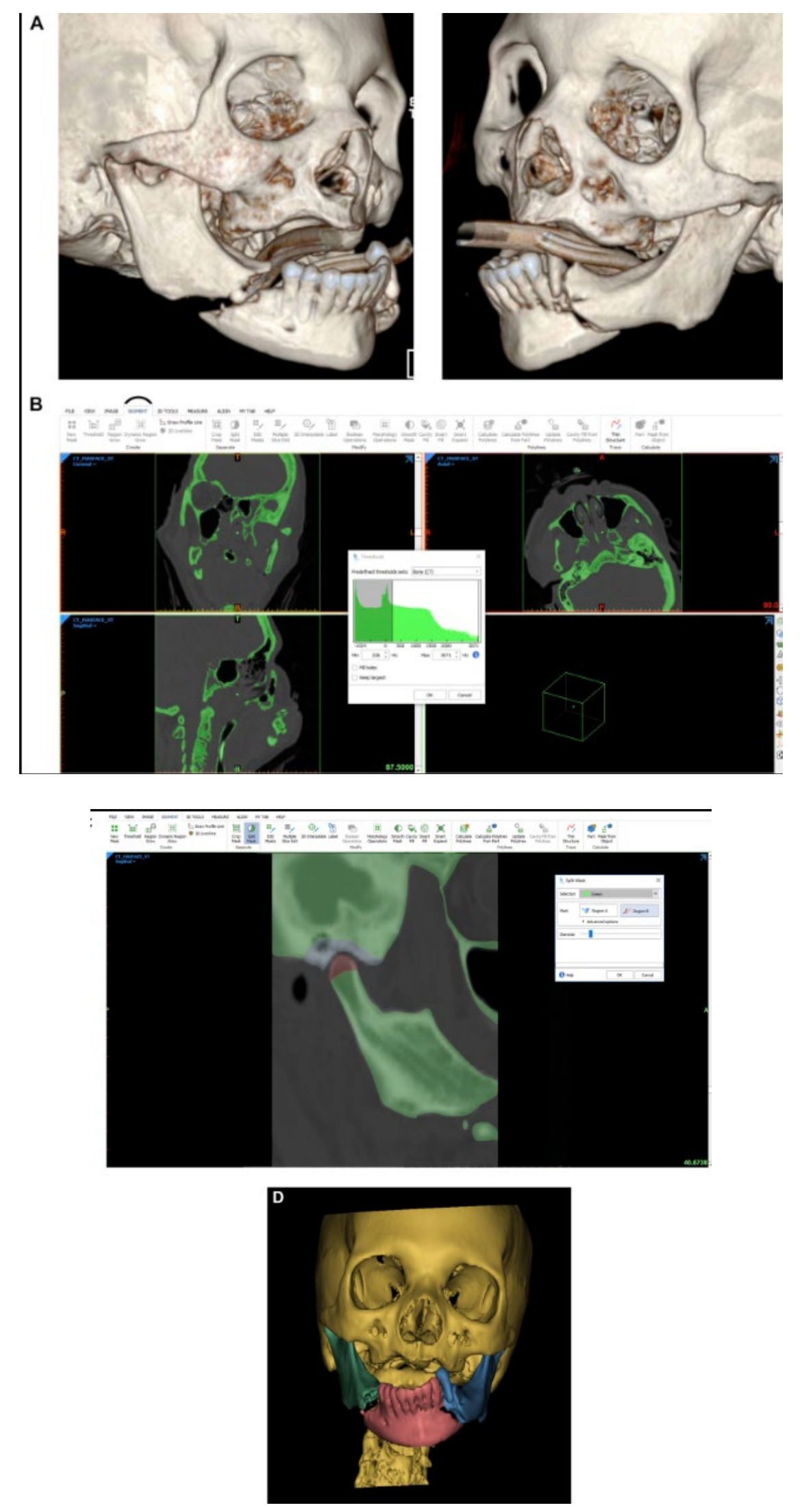


Figure 1. A, Preoperative computed tomography scan with 3-dimensional reconstruction showing bilateral mandible fracture. $B$, When DICOM (Digital Imaging and Communications in Medicine) data have been imported into Mimics, the segmentation tab is clicked and a custom threshold is selected for the patient. $C$, The maxilla and/or mandible and the bone fragments are separated using the split mask function. $D$, By use of the multiple slice edit tool, the segmentation is finished; once this is completed, individual objects of each fracture fragment are created (each fragment is assigned a corresponding color).

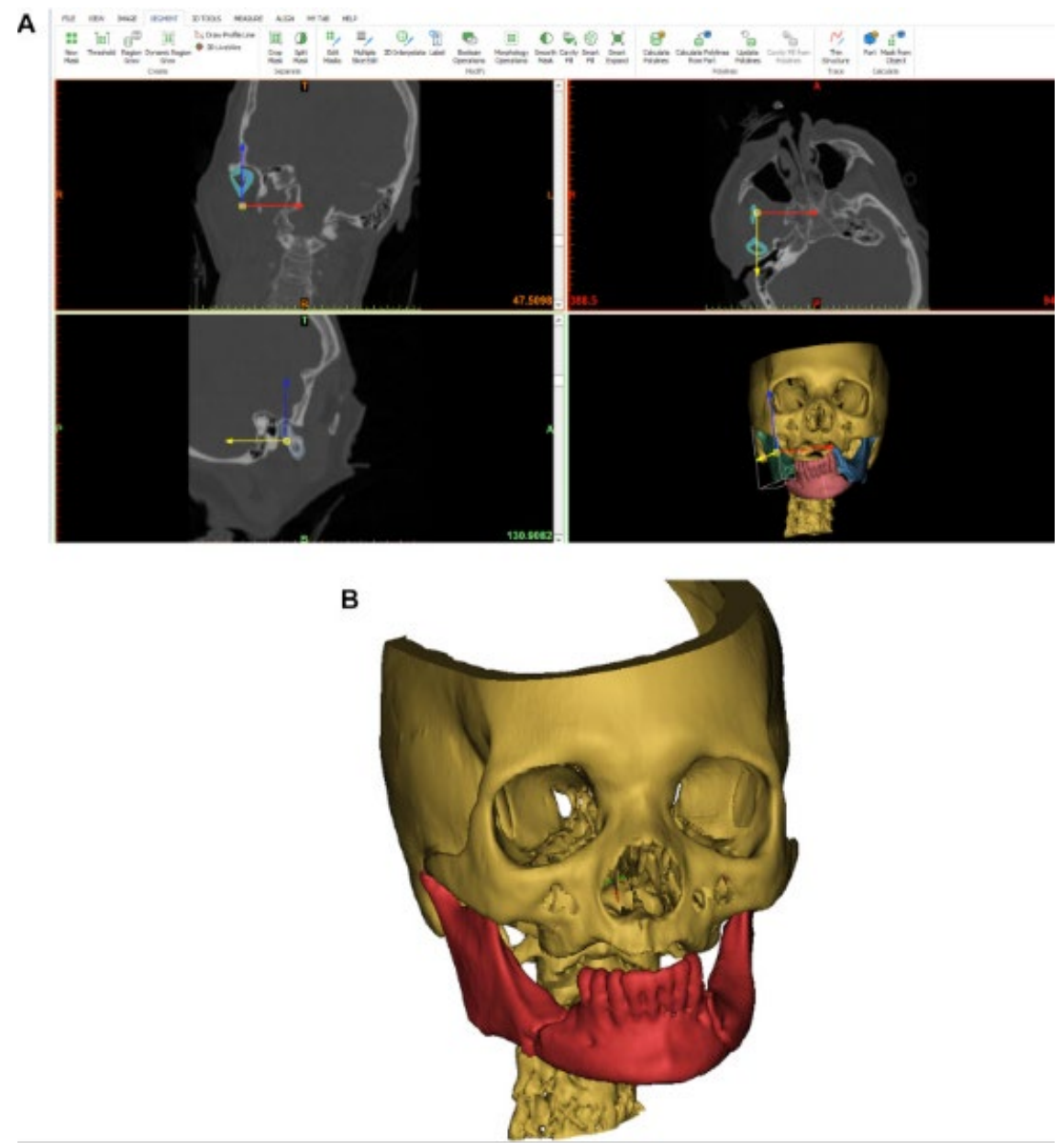




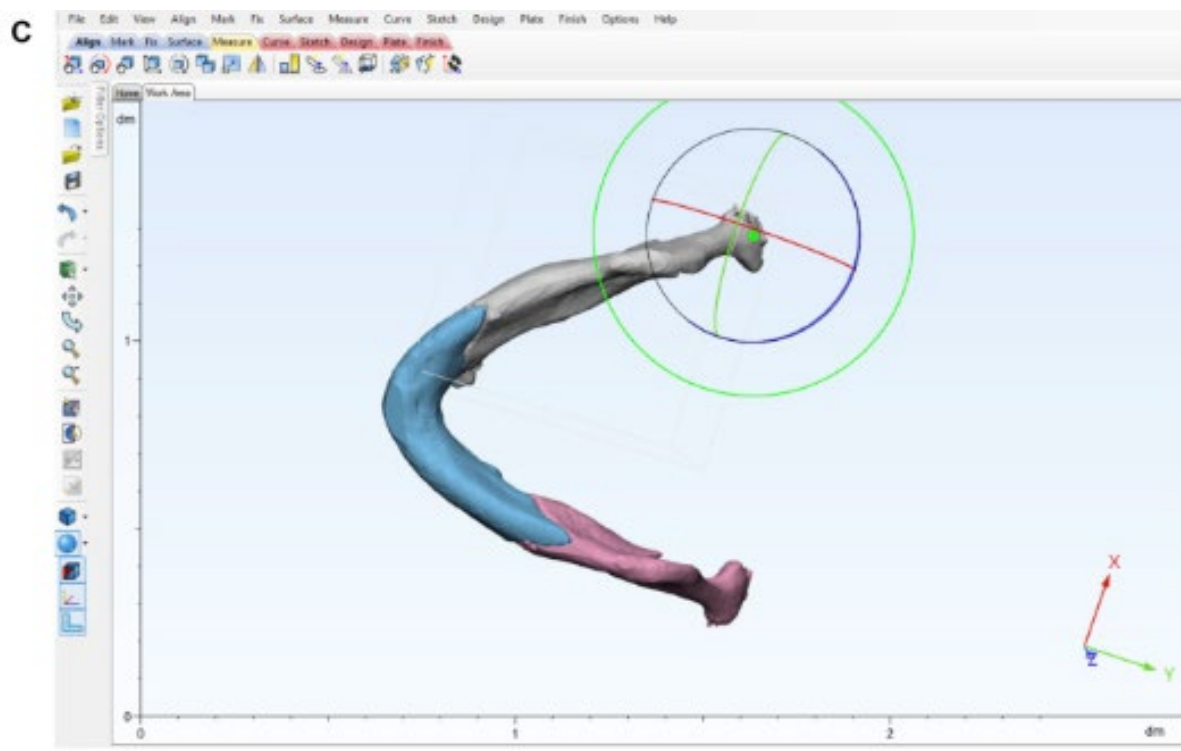

D

Figure 2. A, First, if the mandibular condyles are displaced, the rotate and move tools are used to align the condyles into the mandibular fossa. It is imperative to evaluate the position in axial, coronal, and sagittal planes. $B$, The bone fragments are aligned. If occlusion is present, it can be used as a guide toward virtual reduction. $C$, The objects are imported into 3-matic software as stereolithography (STL) files, and all of the STL files are merged into a single file. $D$, The STL file is imported into the NewPro printing software, and alignment to the print bed is performed, followed by printing.

After virtual reduction is completed, the segmented fragments are transferred as stereolithography (STL) files. STL file objects are imported into 3-matic software (Materialise), whereupon all the STL files are merged into a single file (Fig 2C). Next, the "fix" wizard is run to find errors and fix the STL files. After this digital repair is completed, the file is exported as a binary STL file for $3 D$ printing. The STL file is imported into 
NewPro printing software (NewPro 3D, Vancouver, British Columbia, Canada) (Fig 2D). Last, the virtually reduced mandible is aligned to the print bed, and then supports are generated and printing is performed. White resin (VeriModel OS; Whip Mix, Louisville, KY) was used to print this model. We used the NewPro NP1 prototype printer (NewPro 3D). The total print time was approximately 1 hour, with a post-print wash in fresh isopropyl alcohol for 15 minutes and curing for 15 minutes.

After the virtually reduced mandible was printed, application of the titanium reconstruction plate was completed ( $\mathrm{Fig} 3 \mathrm{~A})$. A senior oral and maxillofacial surgery resident prebent a 2.4-mm titanium locking reconstruction plate (DePuy Synthes, Raynham, MA) to the 3dimensionally printed model of the virtually reduced mandible fracture. This took approximately 7 minutes. The prebent $2.4-\mathrm{mm}$ locking reconstruction plate was then sterilized before surgery. The operation was completed in standard fashion using a transcervical approach. The fit was clinically identical to the virtually reduced and printed model (Fig $3 \mathrm{~B})$. No intraoperative plate manipulation had to be completed. Vivigen cellular bone matrix (DePuy Synthes) was placed around the fracture sites. The OR treatment time was 1.5 hours versus an approximate time of 2.25 hours for traditional ORIF at our institution. A postoperative CT scan was taken ( $\mathrm{Fig} 3 \mathrm{C})$.
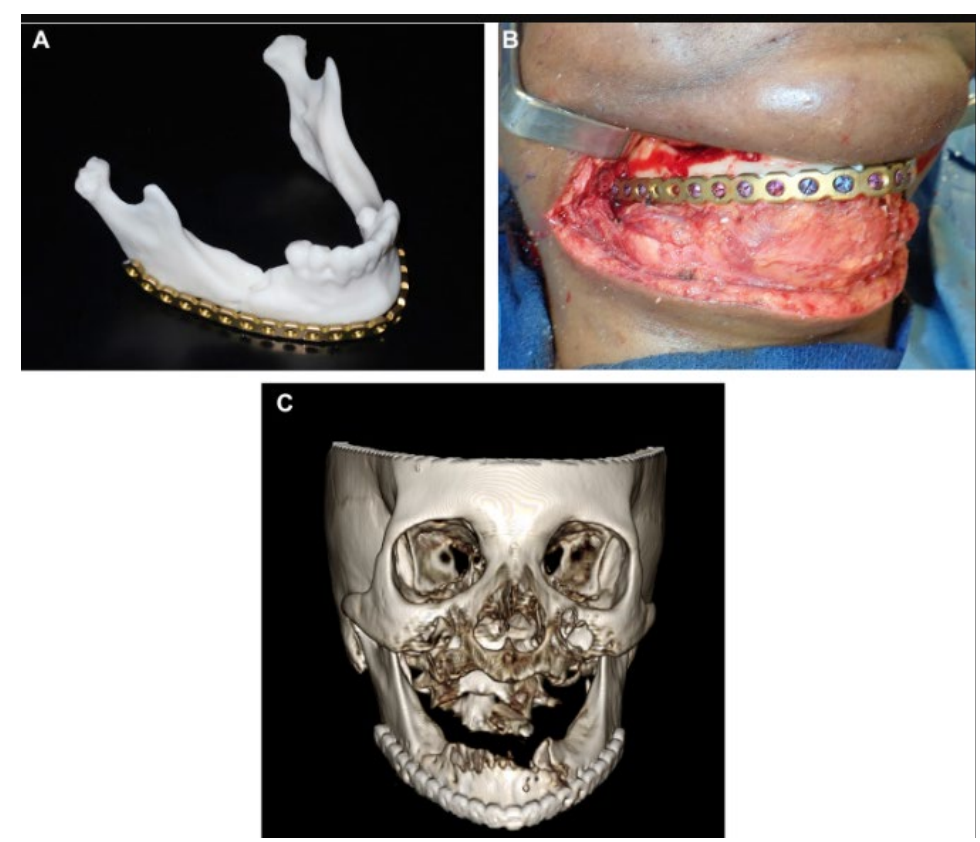
Figure 3. $A$, Three-dimensionally printed model of virtually reduced mandible and ideally contoured titanium reconstruction plate. $B$, Intraoperative plate fixation. $C$, Postoperative computed tomography scan.

\section{DISCUSSION}

CAD-CAM is becoming more commonplace in oral and maxillofacial surgery because it gives surgeons the ability to preplan and enhance execution, yielding better results for patients. Even though CAD-CAM modalities have been around for over a decade, there is still a gap in knowledge of CAD-CAM and its applicability for acute mandibular trauma. This is largely because of 1 - to 2-week lead times from the date of injury to plate delivery because of the multiple steps required, including uploading of CT scans to third parties (eg, Proplan CMF; Synthes, Oberdorf, Switzerland), scheduling an online VSP session, and plate milling and/or shipping. In this article, we demonstrate a novel technique that allows surgeons to virtually reduce complex mandible fractures in-house and subsequently print a model allowing for preoperative plate bending all within a few hours.

Our method used the CT scan of the patient acquired on initial presentation to the emergency department. This scan was transferred to a disc, whereupon the DICOM data were loaded into Mimics software, which is approved by the FDA. A medical-grade CT scan with $1.5-\mathrm{mm}$ cuts is adequate for this technique. These data were then manipulated to facilitate virtual reduction of the patient's complex mandible fracture. After the data were loaded into Mimics, a radiologist and oral and maxillofacial surgeon team completed virtual reduction in approximately 20 minutes. The time required to complete virtual reduction is dependent on the user's familiarity with the software and anatomy. The radiologist in this case was very experienced, which facilitated the time observed in our patient. In our experience, the learning curve to become familiar with the software is not difficult as long as basic principles regarding imaging navigation and thresholding are applied. For those individuals who do not find technology intuitive, understanding the software could be a barrier; however, bioengineers and/or radiologists can complete segmentation. When occlusion is present, the occlusion can be virtually re-established, facilitating reduction. The use of locking reconstruction plates can negate any minor discrepancies that may occur from virtual reductions (based on occlusion or not) that are 
not perfect. Overlaying data obtained from scanning stone models or from intraoral scans will facilitate a much more accurate occlusion; however, this was not completed in our case. After reduction was completed, the data were transferred to the printing software to establish the best printing orientation and number of printing supports. The ability of the $3 \mathrm{D}$ printer used by the surgeon is a bottleneck in this technique. For example, our institution has the NewPro NP1 prototype printer, which is capable of printing a model within 1 hour. This printer is likely not available to most clinicians and has substantial cost. However, more common commercially available printers such as the Form2 (Formlabs, Somerville, MA), which costs approximately $\$ 3,500$, can be used as well, but print times will be approximately 5 to 7 hours. This time difference may not be clinically relevant, as most surgical procedures will take place after this time frame. The 3D print resin (VeriModel OS) we used has a cost of $\$ 175$ for a $1-\mathrm{kg}$ container, which contains $910 \mathrm{~mL}$ of resin. On the basis of our experience, approximately $80 \mathrm{~mL}$ of resin is used per model. This corresponds to an approximate cost of $\$ 15.50$ per model for resin alone. There are also consumables associated with $3 \mathrm{D}$ printing such as the resin tank, printing base, isopropyl alcohol bath, and curing station. A resin tank costs approximately $\$ 100$ and can be used around 10 times. For the isopropyl alcohol bath, we use about $1 \mathrm{~L}$ of isopropyl alcohol per part, at a cost of $\$ 5$ per liter. A curing station costs $\$ 500$. When we add the costs of consumables and computer software, the approximate cost per model is $\$ 50$. Once the mandible is printed, a stock titanium reconstruction plate can be contoured ideally to the model. In this particular case, reconstruction plate contouring took 7 minutes. Another benefit of having a reduced model is that the surgeon now has the ability to analyze the fracture in 3D without soft tissue, which further aids in surgical planning. Once plate bending is completed, the plate can be ready for surgery after sterilization.

Castro-Nuñez et al $\stackrel{12}{2}$ presented 2 cases in which online VSP was used for severely atrophic mandible fractures. In their study, VSP sessions were used to create mirror images of the opposing uninjured mandibular body. Once this was completed, an STL model was fabricated and sent to the authors' institution so that a plate could be contoured before surgery. The turnaround time for the delivery of the model was mentioned in 1 of the 2 cases, which was 5 days. With our technique described in this article, this process 
is streamlined and an ideally virtually reduced model can be ready for plate contouring within hours, not days.

The logical next step using VSP and CAD-CAM principles is having an offsite engineer virtually reduce the fractured mandible and subsequently mill a custom plate. Kokosis et al 4 reported on this technique. In their report, admission CT scans were uploaded to a third party (Proplan CMF) for a VSP session that the surgeons attended. During this session, the patients' occlusion was virtually restored and the fractured segments were subsequently realigned. Of the 5 patients, 3 received custom-milled plates and 2 received prebent plates. The average interval from injury to plate availability was 7 days. Similarly to our report, Kokosis et al virtually reduced the mandible fractures; however, they completed this via a third party, which dramatically slows the process. For example, the surgeon was approving the virtual case plan on post-injury day 3. In comparison, with our technique, an ideally contoured plate can be ready for sterilization in as little as 2 hours after DICOM upload under ideal circumstances. However, we did not use a patientspecific milled plate. In addition, all fractures were approached intraorally, whereas we completed a transcervical approach.

The 2 previously mentioned studies used online methods of VSP and offsite 3D printing and milling; however, office-based methods currently exist. Elegbede et al $\stackrel{10}{ }$ described an office-based workflow for acute mandible fractures. By use of open-source software, mandible fractures were virtually reduced and printed using a commercially available printer, similarly to our technique. However, key differences between the 2 methods exist: First, the open-source software (3D Slicer) used by Elegbede et al is not currently approved by the FDA. In contrast, the software (Mimics and 3-matic) we used is approved. Second, plate contouring was completed intraoperatively by Elegbede et al, negating any potential cost savings from reduced OR time via preoperative plate bending.

King et al11 showed that prebending reconstruction plates before surgery can decrease OR time and thus OR costs. In their report, displaced mandible fractures were reshaped using high-speed drills and reassembled with adhesive under visualization of the fractured segments without the aid of occlusion, in contrast to our virtual reduction, which facilitates no post-print manipulation. The overall time for their workflow is similar to ours. 
To our knowledge, this is the first report of a completely digital workflow using FDAapproved software, with preoperative plate bending that can facilitate a plate ready for sterilization in as little as 2 hours (Fig 4). Although this is a report of a single case, our emphasis is on the description of the digital process. Going forward, this method could potentially be automated via a trained in-house technician, an application, or artificial intelligence. Indeed, this process may one day be accurate enough for the elimination of intraoperative IMF. Further investigation is needed to fully elucidate the cost per case, OR time reduction versus traditional ORIF, and objective accuracy of reduction compared with traditional ORIF. This is currently being investigated at our institution.

\section{In-house digital workflow}

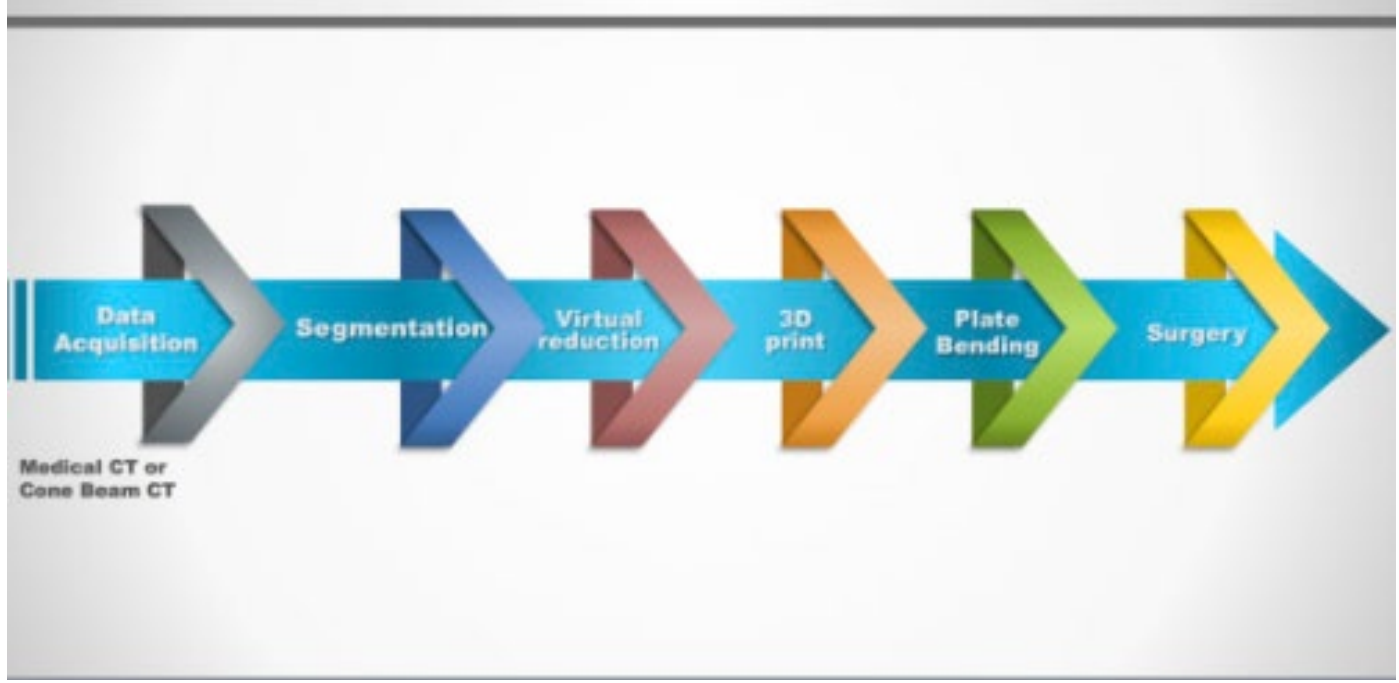

Figure 4. Schema of expedited digital workflow. CT, computed tomography; 3D, 3dimensional.

\section{REFERENCES}

1. Hanasono, M. M., \& Skoracki, R. J. (2013). Computer-assisted design and rapid prototype modeling in microvascular mandible reconstruction. The Laryngoscope, 123(3), 597-604. https://doi.org/10.1002/lary.23717

2. Kim, Y. C., Jeong, W. S., Park, T., Choi, J. W., Koh, K. S., \& Oh, T. S. (2017). The accuracy of patient specific implant prebented with 3D-printed rapid prototype model for 
orbital wall reconstruction. Journal of Cranio-Maxillofacial Surgery, 45(6), 928-936. https://doi.org/10.1016/j.jcms.2017.03.010

3. Miloro, M., \& Markiewicz, M. R. (2017). Virtual Surgical Planning for Inferior Alveolar Nerve Reconstruction. Journal of Oral and Maxillofacial Surgery, 75(11), 2442-2448. https://doi.org/10.1016/j.joms.2017.04.001

4. Kokosis, G., Davidson, E. H., Pedreira, R., Macmillan, A., \& Dorafshar, A. H. (2018). The Use of Computer-Aided Design and Manufacturing in Acute Mandibular Trauma Reconstruction. Journal of Oral and Maxillofacial Surgery, 76(5), 1036-1043. https://doi.org/10.1016/j.joms.2017.12.008

5. Rodby, K. A., Turin, S., Jacobs, R. J., Cruz, J. F., Hassid, V. J., Kolokythas, A., \& Antony, A. K. (2014). Advances in oncologic head and neck reconstruction: Systematic review and future considerations of virtual surgical planning and computer aided design/computer aided modeling. Journal of Plastic, Reconstructive \& Aesthetic Surgery, 67(9), 1171-1185. https://doi.org/10.1016/j.bjps.2014.04.038

6. Swennen, G. R. J., Mollemans, W., \& Schutyser, F. (2009). Three-Dimensional Treatment Planning of Orthognathic Surgery in the Era of Virtual Imaging. Journal of Oral and Maxillofacial Surgery, 67(10), 2080-2092. https://doi.org/10.1016/j.joms.2009.06.007

7. Toto, J. M., Chang, E. I., Agag, R., Devarajan, K., Patel, S. A., \& Topham, N. S. (2015). Improved operative efficiency of free fibula flap mandible reconstruction with patientspecific, computer-guided preoperative planning. Head \& Neck, 37(11), 1660-1664. https://doi.org/10.1002/hed.23815

8. Wagner, J. D., Baack, B., Brown, G. A., \& Kelly, J. (2004). Rapid 3-dimensional prototyping for surgical repair of maxillofacial fractures: A technical note. Journal of Oral and Maxillofacial Surgery, 62(7), 898-901. https://doi.org/10.1016/j.joms.2003.10.011

9. Mazzoni, S., Bianchi, A., Schiariti, G., Badiali, G., \& Marchetti, C. (2015). Computer-Aided Design and Computer-Aided Manufacturing Cutting Guides and Customized Titanium Plates Are Useful in Upper Maxilla Waferless Repositioning. Journal of Oral and Maxillofacial Surgery, 73(4), 701-707. https://doi.org/10.1016/j.joms.2014.10.028

10. Elegbede, A., Diaconu, S. C., McNichols, C. H. L., Seu, M., Rasko, Y. M., Grant, M. P., \& Nam, A. J. (2018). Office-Based Three-Dimensional Printing Workflow for 
Craniomaxillofacial Fracture Repair. Journal of Craniofacial Surgery, 29(5), e440. https://doi.org/10.1097/SCS.0000000000004460

11. King, B. J., Park, E. P., Christensen, B. J., \& Danrad, R. (2018). On-Site 3-Dimensional Printing and Preoperative Adaptation Decrease Operative Time for Mandibular Fracture Repair. Journal of Oral and Maxillofacial Surgery, 76(9), 1950.e1-1950.e8. https://doi.org/10.1016/j.joms.2018.05.009

12. Castro-Núñez, J., Shelton, J. M., Snyder, S., \& Sickels, J. V. (2018). Virtual Surgical Planning for the Management of Severe Atrophic Mandible Fractures. Craniomaxillofacial Trauma \& Reconstruction, 11(2), 150-156. https://doi.org/10.1055/s-0037-1601865 